\title{
SYNTHESIS AND ANTIBACTERIAL ACTIVITY OF NOVEL 5-ARYLIDENE-2-IMINO-3-(2- PHENYL- 1,8-NAPHTHYRIDIN-3-YL)THIAZOLIDIN-4-ONES
}

\author{
RAMALINGAM KUNDENAPALLY ${ }^{1}$, DOMALA RAMESH ${ }^{1 *}$, BATHULA SREENIVASULU ${ }^{2}$
}

\begin{abstract}
${ }^{1}$ Department of Chemistry and Pharmaceutical Sciences, Mahatma Gandhi University, Nalgonda, Telangana, India. ${ }^{2}$ Centre for Chemical Sciences and Technology, Institute of Science and Technology, Jawaharlal Nehru Technological University, Hyderabad, Telangana, India. Email: synchemram@gmail.com
\end{abstract}

Received: 27 May 2019, Revised and Accepted: 21 June 2019

ABSTRACT

Objectives: Nowadays, antimicrobial resistance represents one of the most significant challenges in the medical community. To overcome the problem, it requires the discovery of newer safe and effective molecules against infectious sickness. Synthesis and screening of 1,8-naphthyridines have attracted much attention over the decades since it plays a key role against the microorganisms.

Methods: 1,8-naphthyridine based 5-arylidene derivatives of thiazolidinone (3a-i) has been achieved by the cyclization reaction of 2 -chloro- $N$ (2-phenyl-1,8-naphthyridin-3-yl)acetamide (1) with potassium thiocyanate in acetone followed by its Knoevenagel condensation reaction with appropriate arylaldehydes in ethanol. All the resulting products were confirmed using spectral and physicochemical data. Antibacterial activity was performed against different bacterial strains by agar disc diffusion method using ciprofloxacin as standard.

Results: Compound 3b showed tremendous antibacterial activity among all the tested compounds.

Conclusions: This study provides several advantages such as shorter reaction times, clean product, and good yields. Most of the final products possessed moderate to excellent antibacterial activity.

Keywords: 1,8-Naphthyridine, Thiazolidin-4-one, $N$-2-chloroacetamide, Knoevenagel, Antibacterial activity.

(C) 2019 The Authors. Published by Innovare Academic Sciences Pvt Ltd. This is an open access article under the CC BY license (http://creativecommons. org/licenses/by/4. 0/) DOI: http://dx.doi.org/10.22159/ajpcr.2019.v12i8.34273

\section{INTRODUCTION}

Over the decades, nitrogen and sulfur-containing heterocyclic analog synthesis have been an outstanding area of research due to their profound effect on pharmaceutical applications (Fig. 1). Besides, in the pursuit of the invention of new drugs/chemical entities obtained by the assembly of various bioactive components has gained great attention in modern medicinal chemistry. Consequently, the combination of assorted motifs into one core structure may lead to possessing new modes of therapeutic efficacy [1].

Among the different classes of heterocyclic units, the 1,8-naphthyridine unit is a privileged scaffold and widely distributed in many biologically active synthetic compounds [2]. 1,8-naphthyridine and its derivatives possess valuable pharmacological applications including antibacterial [3], antidepressant [4], antitubercular [5], anticancer [6], antihypertensive [7], and antiplatelet [8] activities. Similarly, thiazolidin-4-one and its 5 -arylidene derivatives are represented as an interesting class of molecules showing a broad-spectrum of medical applications such as antimicrobial [9], anti-inflammatory [10], DPPH radical scavenger [11], antifungal [12], anticancer [13], and anticonvulsant [14] activities.

Thiazolidin-4-one ring construction is well documented $[15,16]$, but this type of cyclization is not much explored on the 1,8-naphthyridine nucleus. By considering afore-mentioned findings and continuation of our research on 1,8-naphthyridines [17], we herein report the synthesis of novel 5-arylidene-2-imino-3-(2-phenyl-1,8-naphthyridin3-yl)thiazolidin-4-ones and its biological screening were evaluated.

\section{MATERIALS AND METHODS}

Materials

All reagents were used as purchased from commercial sources and were used without any further purification.

\section{Methods}

Synthesisof2-imino-3-(2-phenyl-1,8-naphthyridin-3-yl)thiazolidin-4-one(2) 2-chloro- $N$-(2-phenyl-1,8-naphthyridin-3-yl)acetamide [18] (1) ( $3 \mathrm{mmol}$ ) and potassium thiocyanate $(6 \mathrm{mmol})$ were dissolved in anhydrous acetone $(30 \mathrm{~mL})$ to give a clear solution and refluxed for $3 \mathrm{~h}$ till completion of the reaction (TLC monitored). After that, the solvent was evaporated and obtained solid was washed with water $(50 \mathrm{~mL})$, filtered and dried. The residue was subjected to flash chromatography (ethyl acetate-hexane 2:8 v/v) to get the compound 2 as white solid. Yield 72\%; mp: $228^{\circ} \mathrm{C}$; IR ( $\left.\mathrm{KBr}, \mathrm{cm}^{-1}\right)$ : $3284(\mathrm{NH}), 1720(\mathrm{C}=0), 1685$, 1616, 1544, 698; ${ }^{1} \mathrm{H}$ NMR (400 MHz, DMSO- $\left.d_{6}\right) \delta(\mathrm{ppm}): 3.81-4.05(2 \mathrm{H}$, $\left.\mathrm{dd}, J=55.16,17.11 \mathrm{~Hz}, \mathrm{CH}_{2}\right), 7.42-7.70(7 \mathrm{H}, \mathrm{m}), 8.30-8.41(2 \mathrm{H}, \mathrm{m})$, $9.19(1 \mathrm{H}, \mathrm{s}) ;{ }^{13} \mathrm{CNMR}\left(100 \mathrm{MHz}, \mathrm{DMSO}-d_{6}\right) \delta(\mathrm{ppm}): 33.74\left(\mathrm{CH}_{2}\right), 121.71$, $122.35,127.43,127.85,128.03,129.08,137.01,137.26,138.94,154.61$, 154.94, 159.67, 160.61, $170.48(\mathrm{C}=0)$; MS: $[\mathrm{M}+1]^{+}=321$. Anal. Calc. for $\mathrm{C}_{17} \mathrm{H}_{12} \mathrm{~N}_{4} \mathrm{OS}$ : C, 63.73; H, 3.78; N, 17.49; 0, 4.99; S, 10.01; Found: C, 63.75; H, 3.82; N, 17.51; $0,5.02 ; \mathrm{S}, 10.04$.

Synthesis of 5-arylidene-2-imino-3-(2-phenyl-1,8-naphthyridin-3-yl) thiazolidin-4-one derivatives (3a-i)

A mixture of compound 2 (1 mmol), substituted benzaldehyde ( $1 \mathrm{mmol})$, and piperidine (2-3 drops) in ethanol $(20 \mathrm{~mL})$ were stirred under reflux conditions for 6-8 h. After the completion of reaction (TLC monitored), cool and diluted with ice-water $(30 \mathrm{~mL})$. The solid was separated by filtration, air-dried and recrystallized from $96 \%$ ethanol gave the compounds $3 \mathrm{a}-\mathrm{h}$.

5-Benzylidene-2-imino-3-(2-phenyl-1,8-naphthyridin-3-yl)thiazolidin-4one (3a)

Light brown solid; yield:82\%; mp:226-228 ${ }^{\circ} \mathrm{C}$; IR $\left(\mathrm{KBr}, \mathrm{cm}^{-1}\right): 3365$ (NH), 1720 (C=0), 1649, 1604, 1544, 1427, 694 $\mathrm{cm}^{-1}$; ${ }^{1} \mathrm{H}$ NMR (300 MHz, 


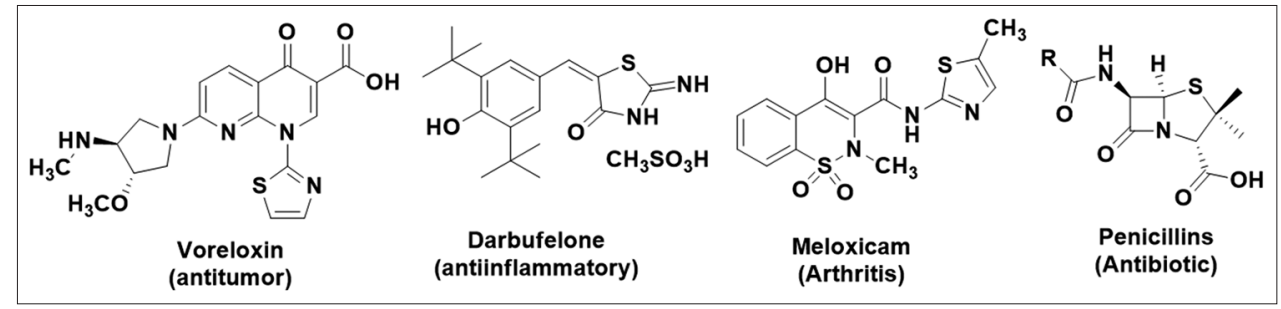

Fig. 1: Chemical structures of biologically active compounds

$\left.\mathrm{CDCl}_{3}\right) \delta(\mathrm{ppm}): 7.38-7.51(8 \mathrm{H}, \mathrm{m}), 7.66-7.78(3 \mathrm{H}, \mathrm{dd}, J=13.89,8.99 \mathrm{~Hz})$, $7.94(2 \mathrm{H}, \mathrm{s}), 8.11-8.29(2 \mathrm{H}, \mathrm{m}), 9.07-9.09(1 \mathrm{H}, \mathrm{d}, J=4.26 \mathrm{~Hz}) ;{ }^{13} \mathrm{C}$ NMR $\left(100 \mathrm{MHz}, \mathrm{CDCl}_{3}\right) \delta(\mathrm{ppm}): 121.11,121.53,122.25,122.35,126.85$, $128.01,128.22,128.45,129.16,129.39,129.91,130.18,130.32,132.36$, $136.32,140.68,152.88,153.92,156.95,162.80,167.30$ (C=0); MS: $[\mathrm{M}+1]^{+}=409$; Anal. Calc. for $\mathrm{C}_{24} \mathrm{H}_{16} \mathrm{~N}_{4} \mathrm{OS}$ : C, 70.57; H, 3.95; N, 13.72; O, 3.92; S, 7.85; Found: C, 70.62; H, 3.98; N, 13.75; 0, 3.96; S, 7.89.

5-(4-Chlorobenzylidene)-2-imino-3-(2-phenyl-1,8-naphthyridin-3-yl) thiazolidin-4-one $(3 b)$

Pale yellow solid; yield:79\%; mp:241-243 ${ }^{\circ} \mathrm{C}$; IR $\left(\mathrm{KBr}, \mathrm{cm}^{-1}\right)$ : 3365 (NH), 1712 (C=0), 1647, 1602, 1520, 1489, 1340, 692; ${ }^{1} \mathrm{H}$ NMR (300 $\left.\mathrm{MHz}, \mathrm{CDCl}_{3}\right) \delta(\mathrm{ppm}): 7.29-7.54(9 \mathrm{H}, \mathrm{m}), 7.66(1 \mathrm{H}, \mathrm{s},=\mathrm{NH}), 7.76(1 \mathrm{H}, \mathrm{s},-$ $\mathrm{C}=\mathrm{CH}), 7.92-7.97(2 \mathrm{H}, \mathrm{d}, J=4.11 \mathrm{~Hz}), 8.17-8.23(1 \mathrm{H}, \mathrm{d}, J=7.89 \mathrm{~Hz}), 9.10-$ $9.12(1 \mathrm{H}, \mathrm{d}, J=4.21 \mathrm{~Hz}) ;{ }^{13} \mathrm{C}$ NMR $\left(75 \mathrm{MHz}, \mathrm{CDCl}_{3}\right) \delta(\mathrm{ppm}): 122.77127 .30$, $128.93,129.26,129.38,129.52,131.44,132.02,134.55,137.63,138.40$, 141.30, 151.68, 151.98, 152.54, 156.38, 167.21 (C=0); MS: $[\mathrm{M}+1]^{+}=443$; Anal. Calc. for $\mathrm{C}_{24} \mathrm{H}_{15} \mathrm{~N}_{4} \mathrm{OSCl}$ : C, 65.08; $\mathrm{H}, 3.41 ; \mathrm{Cl}, 8.00 ; \mathrm{N}, 12.65 ; 0,3.61$; S, 7.24; Found: C, 65.12; H, 3.45; Cl, 8.03; N, 12.70; 0, 3.62; S, 7.26.

5-(2-Chlorobenzylidene)-2-imino-3-(2-phenyl-1,8-naphthyridin-3-yl) thiazolidin-4-one (3c)

Pale yellow solid; yield:77\%; mp:255-257 ${ }^{\circ}$; IR $\left(\mathrm{KBr}, \mathrm{cm}^{-1}\right): 3290(\mathrm{NH})$, 1711 (C=0), 1652, 1612, 1538, 1435, 691; ${ }^{1} \mathrm{H}$ NMR (300 MHz, DMSO- $d$ ) $\delta(\mathrm{ppm}): 7.24-7.55(8 \mathrm{H}, \mathrm{m}), 7.69(1 \mathrm{H}, \mathrm{s},=\mathrm{NH}), 7.87(1 \mathrm{H}, \mathrm{s},-\mathrm{C}=\mathrm{CH}), 8.09-$ $8.12(2 \mathrm{H}, \mathrm{d}, J=4.10 \mathrm{~Hz}), 8.19-8.28(2 \mathrm{H}, \mathrm{m}), 8.77-8.79(1 \mathrm{H}, \mathrm{d}, J=4.31 \mathrm{~Hz})$; ${ }^{13} \mathrm{C}$ NMR $\left(75 \mathrm{MHz}\right.$, DMSO- $\left.d_{6}\right) \delta(\mathrm{ppm}): 121.67,122.20,124.94,125.94$, $127.12,127.75,128.25,128.89,129.25,129.63,130.06,130.44,132.75$, 133.78, 134.97, 151.82, 152.46, 153.69, 159.38, 168.29 (C=0). MS: $[\mathrm{M}+1]^{+}=443$; Anal. Calc. for $\mathrm{C}_{24} \mathrm{H}_{15} \mathrm{ClN}_{4} \mathrm{OS}$ : C, 65.08; $\mathrm{H}, 3.41 ; \mathrm{Cl}, 8.00 ; \mathrm{N}$, 12.65; 0, 3.61; S, 7.24; Found: C, 65.11; H, 3.44; Cl, 8.02; N, 12.69; O, $3.63 ;$ S, 7.28 .

2-Imino-5-(4-methoxybenzylidene)-3-(2-phenyl-1,8-naphthyridin-3-yl) thiazolidin-4-one (3d)

Pale yellow solid; yield:81\%; mp:262-264 ${ }^{\circ} \mathrm{C}$; IR $\left(\mathrm{KBr}, \mathrm{cm}^{-1}\right): 3309(\mathrm{NH})$, 1718 (C=0), 1631, 1618, 1538, 1419, 692; ${ }^{1} \mathrm{H}$ NMR (300 MHz, DMSO- $d_{6}$ ) $\delta(\mathrm{ppm}): 3.78\left(3 \mathrm{H}, \mathrm{s},-\mathrm{OCH}_{3}\right), 7.06-7.09(2 \mathrm{H}, \mathrm{m}), 7.39-7.64(8 \mathrm{H}, \mathrm{m})$, $7.75(1 \mathrm{H}, \mathrm{s},-\mathrm{C}=\mathrm{CH}), 8.09-8.22(3 \mathrm{H}, \mathrm{m}), 8.76-8.79(1 \mathrm{H}, \mathrm{d}, J=4.22 \mathrm{~Hz})$; ${ }^{13} \mathrm{C}$ NMR $\left(100 \mathrm{MHz}, \mathrm{DMSO}-d_{6}\right): 55.31\left(\mathrm{OCH}_{3}\right), 121.67,122.20,125.94$, $128.25,128.89,129.32,129.63,130.06,131.84,132.31,132.75,134.97$, 151.82, 152.46, 153.69, 160.76, 161.38, $167.56(\mathrm{C}=0)$; $\mathrm{MS}:[\mathrm{M}+1]^{+}=439$; Anal. Calc. for $\mathrm{C}_{25} \mathrm{H}_{18} \mathrm{~N}_{4} \mathrm{O}_{2} \mathrm{~S}$ : C, 68.48; H, 4.14; N, 12.78; 0, 7.30; S, 7.31; found: C, 68.52; H, 4.17; N, 12.80; 0, 7.33; S, 7.32.

5-(2,4-Dimethoxybenzylidene)-2-imino-3-(2-phenyl-1,8-naphthyridin-3yl) thiazolidin-4-one (3e)

Yellow orange solid; yield:82\%; mp:196-198 ${ }^{\circ} \mathrm{C}$; IR $\left(\mathrm{KBr}, \mathrm{cm}^{-1}\right): 3128$ $(\mathrm{NH}), 1708$ (C=0), 1635, 1587, 1500, 1460, 694; ${ }^{1} \mathrm{H}$ NMR (300 MHz, $\left.\mathrm{CDCl}_{3}\right) \delta(\mathrm{ppm}): 3.82\left(\mathrm{~d}, 6 \mathrm{H}, J=12.04 \mathrm{~Hz}, 2-\mathrm{OCH}_{3}\right), 6.42-6.46(2 \mathrm{H}, \mathrm{m})$, $7.20(1 \mathrm{H}, \mathrm{d}, J=8.62 \mathrm{~Hz}), 7.40-7.49(5 \mathrm{H}, \mathrm{m}), 7.77(1 \mathrm{H}, \mathrm{s},-\mathrm{C}=\mathrm{CH})$, 7.94-7.97 (2H, d, J=3.71 Hz), 8.09-8.18 $(2 \mathrm{H}, \mathrm{m}), 9.08-9.12(1 \mathrm{H}, \mathrm{d}$, $J=5.64 \mathrm{~Hz}) ;{ }^{13} \mathrm{C}$ NMR $\left(75 \mathrm{MHz}, \mathrm{CDCl}_{3}\right) \delta(\mathrm{ppm}): 55.47,55.88\left(2-\mathrm{OCH}_{3}\right)$, $98.47,106.32,114.32,118.70,124.66,128.02,129.22,129.47,129.60$, $137.65,137.95,141.32,152.15,153.06,156.25,159.54,162.63,167.40$ $(\mathrm{C}=0), \mathrm{MS}:[\mathrm{M}+1]^{+}=469$; Anal. Calc. for $\mathrm{C}_{26} \mathrm{H}_{20} \mathrm{~N}_{4} \mathrm{O}_{3} \mathrm{~S}: \mathrm{C}, 66.65 ; \mathrm{H}, 4.30 ; \mathrm{N}$,
11.96; 0, 10.24; S, 6.84; found: C, 66.68; H, 4.32; N, 12.00; 0, 10.28; S, 6.87.

2-Imino-5-(4-nitrobenzylidene)-3-(2-phenyl-1,8-naphthyridin-3-yl) thiazolidin-4-one ( $3 \mathrm{f}$ )

Light yellow solid; yield:80\%; mp:248-250 ${ }^{\circ} \mathrm{C}$; $\left(\mathrm{KBr}, \mathrm{cm}^{-1}\right)$ : 3324 (NH), 1719 (C=0), 1640, 1575, 1521, 698; ${ }^{1} \mathrm{H}$ NMR (300 MHz, $\mathrm{CDCl}_{3}$ ) $\delta(\mathrm{ppm}): \delta 7.38-7.54(9 \mathrm{H}, \mathrm{m}), 7.64(1 \mathrm{H}, \mathrm{s},=\mathrm{NH}), 7.90(1 \mathrm{H}, \mathrm{s},-\mathrm{C}=\mathrm{CH})$, $7.96-8.12(2 \mathrm{H}, \mathrm{m}), 8.17-8.21(1 \mathrm{H}, \mathrm{d}, J=7.82 \mathrm{~Hz}), 9.10-9.12(1 \mathrm{H} \mathrm{d}$, $J=4.80 \mathrm{~Hz}) ;{ }^{13} \mathrm{C}$ NMR $\left(75 \mathrm{MHz}, \mathrm{CDCl}_{3}\right): 122.75127 .32,128.84,129.35$, $129.36,129.43,131.49,132.11,134.26,137.69,138.32,141.45,151.75$, 151.94, 152.47, 156.69, $167.09(\mathrm{C}=0)$; $\mathrm{MS}:[\mathrm{M}+1]^{+}=454$; Anal. Calc. for $\mathrm{C}_{24} \mathrm{H}_{15} \mathrm{~N}_{5} \mathrm{O}_{3} \mathrm{~S}$ : C, 63.57; H, 3.33; N, 15.44; 0, 10.58; S, 7.07; Found: C, 63.62; H, 3.36; N, 15.48; O, 10.60; S, 7.10

2-Imino-5-(2-nitrobenzylidene)-3-(2-phenyl-1,8-naphthyridin-3-yl) thiazolidin-4-one $(3 g)$

Pale yellow solid; yield:77\%; mp:190-192 ${ }^{\circ} \mathrm{C}$; IR $\left(\mathrm{KBr}, \mathrm{cm}^{-1}\right)$ : $3299(\mathrm{NH})$, 1713 (C=0), 1641, 1609, 1532, 1430, 695; ${ }^{1} \mathrm{H}$ NMR (300 MHz, DMSO- $d_{6}$ ) $\delta$ (ppm): 7.39-7.57 $(5 \mathrm{H}, \mathrm{m}), 7.60(1 \mathrm{H}, \mathrm{s},=\mathrm{NH}), 7.66-7.75(2 \mathrm{H}, \mathrm{m})$, $7.81(1 \mathrm{H}, \mathrm{s},-\mathrm{C}=\mathrm{CH}), 7.86-8.13(4 \mathrm{H}, \mathrm{m}), 8.19-8.22(1 \mathrm{H}, \mathrm{d}, J=7.5 \mathrm{~Hz}), 9.11-$ $9.13(1 \mathrm{H}, \mathrm{d}, J=4.93 \mathrm{~Hz}) ;{ }^{13} \mathrm{C}$ NMR $\left(75 \mathrm{MHz}\right.$, DMSO- $\left.d_{6}\right) \delta(\mathrm{ppm}): 121.67$, $122.20,125.57,125.94,127.98,128.25,128.89,129.63,129.76,130.06$, $130.52,130.85,132.34,132.75,134.97,148.88,151.82,152.46,153.69$, 160.38, $170.17(\mathrm{C}=0)$; $\mathrm{MS}:[\mathrm{M}+1]^{+}=454$; Anal. Calc. for $\mathrm{C}_{24} \mathrm{H}_{15} \mathrm{~N}_{5} \mathrm{O} \mathrm{S}$ : C, 63.57; H, 3.33; N, 15.44; 0, 10.58; S, 7.07; found: C, 63.62; H, 3.35; N, $15.48 ; 0,10.60 ; S, 7.09$.

\section{2-Imino-5-(4-methylbenzylidene)-3-(2-phenyl-1,8-naphthyridin-3-yl)} thiazolidin-4-one (3h)

Pale yellow solid; yield:78\%; mp:207-209 ${ }^{\circ}$; $\left(\mathrm{KBr}_{\mathrm{cm}} \mathrm{cm}^{-1}\right): 3233(\mathrm{NH})$, 2912, 1705 (C=0), 1634, 1580, 1529, 692; ${ }^{1} \mathrm{H}$ NMR (300 MHz, $\left.\mathrm{CDCl}_{3}\right) \delta$ (ppm): $2.43(3 \mathrm{H}, \mathrm{s}) 7.35-7.52(9 \mathrm{H}, \mathrm{m}), 7.65(1 \mathrm{H}, \mathrm{s},=\mathrm{NH}), 7.89-7.93(2 \mathrm{H}$, d, $J=3.54 \mathrm{~Hz}), 8.05(1 \mathrm{H}, \mathrm{s}), 8.12-8.16(1 \mathrm{H}, \mathrm{d}, J=7.49 \mathrm{~Hz}), 9.09-9.11(1 \mathrm{H}$, d, $J=4.94 \mathrm{~Hz}) ;{ }^{13} \mathrm{C}$ NMR $\left(75 \mathrm{MHz}, \mathrm{CDCl}_{3}\right) \delta(\mathrm{ppm}): 21.9\left(\mathrm{CH}_{3}\right), 122.64$, 122.53 127.26, 128.75, 129.14, 129.85, 130.44, 131.88, 133.32, 134.34, $138.67,139.17,142.08,151.90,152.83,153.45,155.72,167.23$ (C=0); MS: $[\mathrm{M}+1]^{+}=423$; Anal. Calc. for $\mathrm{C}_{25} \mathrm{H}_{18} \mathrm{~N}_{4} \mathrm{OS}$ : C, 71.07; $\mathrm{H}, 4.29 ; \mathrm{N}, 13.26$; $0,3.79$; S, 7.59; found: C, 71.11; H, 4.33; N, 13.28; 0, 3.82; S, 7.61.

5-(4-Hydroxybenzylidene)-2-imino-3-(2-phenyl-1,8-naphthyridin-3-yl) thiazolidin -4-one (3i)

IR (KBr, cm${ }^{-1}$ ): $3271(\mathrm{NH}), 1711$ (C=0), 1628, 1614, 1523, 1422, 698; ${ }^{1} \mathrm{H}$ NMR $\left(300 \mathrm{MHz}\right.$, DMSO- $\left.d_{6}\right) \delta(\mathrm{ppm}): 6.93-6.97(1 \mathrm{H}, \mathrm{m}), 7.39-7.59$ $(6 \mathrm{H}, \mathrm{m}), 7.67(1 \mathrm{H}, \mathrm{s}), 7.88(1 \mathrm{H}, \mathrm{s}), 8.09-8.13(2 \mathrm{H}, \mathrm{m}), 8.19-8.34(3 \mathrm{H}, \mathrm{m})$, $8.77-8.79(1 \mathrm{H}, \mathrm{d}, J=3.8), 8.94-8.99(1 \mathrm{H}, \mathrm{d}, J=5.41) ;{ }^{13} \mathrm{C}$ NMR $(100 \mathrm{MHz}$, DMSO- $d$ ) $\delta(\mathrm{ppm}): 116.49,121.67,122.20,125.94,127.92,128.25$, $128.89,129.63,130.06,132.26,132.39,132.75,134.97,151.82,152.46$, 153.69, 159.43, 160.38, $167.18(\mathrm{C}=0)$; MS: $[\mathrm{M}+1]^{+}=425$; Anal. Calc. for $\mathrm{C}_{24} \mathrm{H}_{16} \mathrm{~N}_{4} \mathrm{O}_{2} \mathrm{~S}: \mathrm{C}, 67.91 ; \mathrm{H}, 3.80 ; \mathrm{N}, 13.20 ; \mathrm{O}, 7.54 ; \mathrm{S}, 7.55$; found: C, 67.95; H, 3.83; N, 13.24; O, 7.57; S, 7.58.

\section{RESULTS AND DISCUSSION}

A two-step reaction protocol for the synthesis of novel series of compounds 3a-i is executed in Scheme 1. In the first step, the reaction 
of 2-chloro- $N$-(2-phenyl-1,8-naphthyridin-3-yl)acetamide (1) was performed with KSCN in dry acetone under reflux conditions led to the formation of cyclic product 2-imino-3-(2-phenyl-1,8-naphthyridin-3yl)thiazolidin-4-one (2) exclusively in good yield. This heterocyclization involves the thiocyanation of $\mathrm{N}$-2-haloacetamide (2) to afford a transition intermediate (I) and followed by in situ intramolecular ring closure (Fig. 2). In the second step, the compound (2) was separately condensed with commercially available appropriate benzaldehydes in the presence of piperidine (Knoevenagel condensation) under reflux conditions in ethanol to provide the corresponding target products (3a-i) in excellent yields. All the synthetic compounds ( 2 and 3a-i) were assessed in this study by physical, spectral, and analytical data.

The IR spectra of compound 2 showed absorption bands at 3284 and $1720 \mathrm{~cm}^{-1}$ corresponding to imino $(\mathrm{C}=\mathrm{NH})$ and carbonyl $(\mathrm{C}=0)$ groups, respectively. The ${ }^{1} \mathrm{H}$-NMR spectra of compound 2 revealed that the appearance of a doublet of doublet at the region of 3.81-4.05 ppm corresponding to the methylene protons $\left(\mathrm{CH}_{2}\right.$, thiazolidinone ring) due to deshielded by the adjacent sulfur atom and carbonyl group. The singlet signal observed at $7.60 \mathrm{ppm}$ region $\left(\mathrm{D}_{2} \mathrm{O}\right.$ exchangeable $)$ is related to imine proton. The signals appeared at the region 7.53-9.07 ppm corresponds to aromatic protons of phenyl and 1,8-naphthyridine system. In ${ }^{13} \mathrm{C}-\mathrm{NMR}$, the peas appeared at $33.74,159.67$, and $170.48 \mathrm{ppm}$ region for active methylene $\left(\mathrm{CH}_{2}\right)$, imino $(\mathrm{C}=\mathrm{NH})$, and carbonyl $(\mathrm{C}=\mathrm{O})$ functions, respectively.

IR spectra of 3a-i were obtained at the region of $3365-3130 \mathrm{~cm}^{-1}$ and $1720-1705 \mathrm{~cm}^{-1}$, indicated the frequencies of $\mathrm{NH}$ and carbonyl
$(\mathrm{C}=0)$ functions, respectively. The arylidene compounds $3 \mathrm{a}-\mathrm{i}$ can exist as geometrical $E$ and $Z$ forms (Fig. 3). The ${ }^{1} \mathrm{H}$-NMR spectra showed a new signal at the region of 7.76-7.95 ppm was attributed to methine proton $(-\mathrm{C}=\mathrm{C}-\mathrm{H})$ in $3 \mathrm{a}-\mathrm{i}$. This was the higher chemical shift value than expected values, due to the deshielding effect of an adjacent carbonyl function. Accordingly, the exocyclic $\mathrm{C}=\mathrm{C}$ bond was assigned $Z$-configuration $[19,20]$. In ${ }^{13} \mathrm{C}-\mathrm{NMR}$, methine carbons $(=\mathrm{CH})$ of $3 \mathrm{a}-\mathrm{i}$ appeared at the region of 120.58-129.34 ppm. In mass spectra, all the compounds furnished the corresponding molecular ion peaks, which were matched with the calculated molecular weight, respectively.

\section{Antibacterial activity}

From Table.1, the biological screening data of all the compounds revealed that most of the compounds displayed moderate to good bacterial inhibition. The screening studies indicated that the compounds $3 \mathrm{~b}, 3 \mathrm{~d}$, and $3 e$ were displayed excellent antibacterial activity against bacterial strains and $3 \mathrm{~b}$ was the most promising analog as compared to the ciprofloxacin as a reference drug.

\section{Biological assay}

Antibacterial activity of the final analogs (3a-i) was performed against a panel of bacteria such as Staphylococcus aureus, Bacillus cereus, Escherichia coli, and Pseudomonas aeruginosa by agar disc diffusion method [21] at $50 \mu \mathrm{g} / \mathrm{mL}$ concentration in DMSO as a solvent. The cultures were diluted with $5 \%$ autoclaved saline and adjust the final volume concentration to $\sim 10^{5}-10^{6} \mathrm{CFU} / \mathrm{mL}$. Then, the liquid form of the tested compound was soaked on to a disc $(5 \mathrm{~mm})$. After drying the discs, they were introduced on to the upper layer of medium evenly

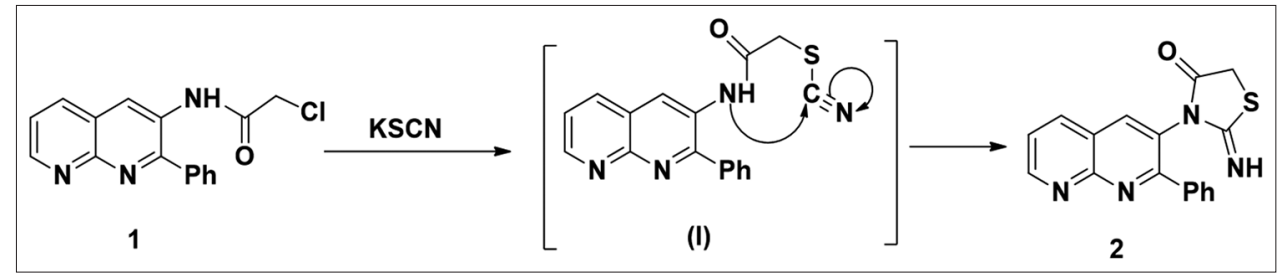

Fig. 2: Mechanistic pathway for compound 2

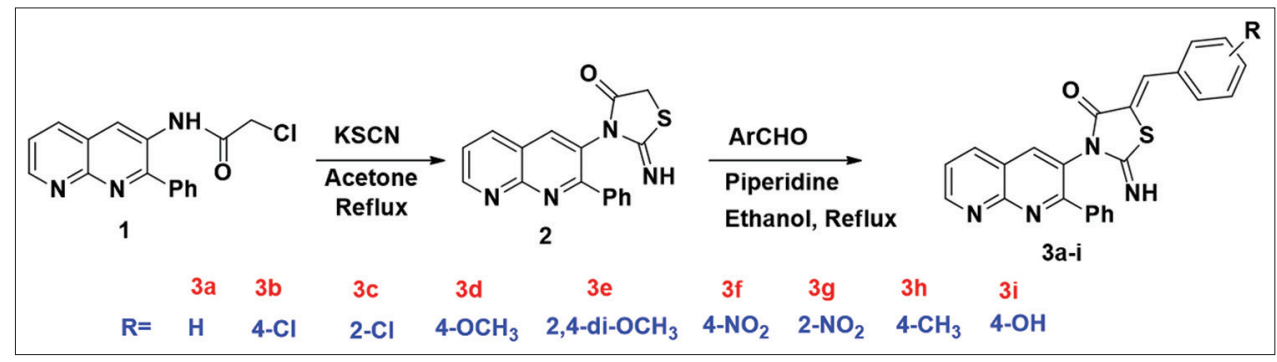

Scheme 1: Synthetic protocol for the final analogs 3a-i

Table 1: Biological activity of the synthetic compounds against the microorganisms

\begin{tabular}{|c|c|c|c|c|}
\hline \multirow[t]{3}{*}{ Entry } & \multicolumn{4}{|c|}{ Microorganism inhibition zone diameter (mm) } \\
\hline & \multicolumn{2}{|l|}{ Gram-positive bacteria } & \multicolumn{2}{|c|}{ Gram-negative bacteria } \\
\hline & Staphylococcus aureus & Bacillus cereus & Escherichia coli & Pseudomonas aeruginosa \\
\hline $3 a$ & 20 & 18 & 07 & 25 \\
\hline $3 b$ & 28 & 29 & 28 & 27 \\
\hline $3 c$ & 23 & 20 & 11 & 16 \\
\hline $3 d$ & 26 & 25 & 23 & 23 \\
\hline $3 f$ & 08 & 12 & 07 & 10 \\
\hline $3 g$ & 12 & 28 & 05 & 16 \\
\hline $3 \mathrm{~h}$ & 25 & 18 & 25 & 26 \\
\hline $3 \mathrm{i}$ & 11 & 13 & 09 & 14 \\
\hline Ciprofloxacin (std.) & 22 & 24 & 24 & 23 \\
\hline
\end{tabular}




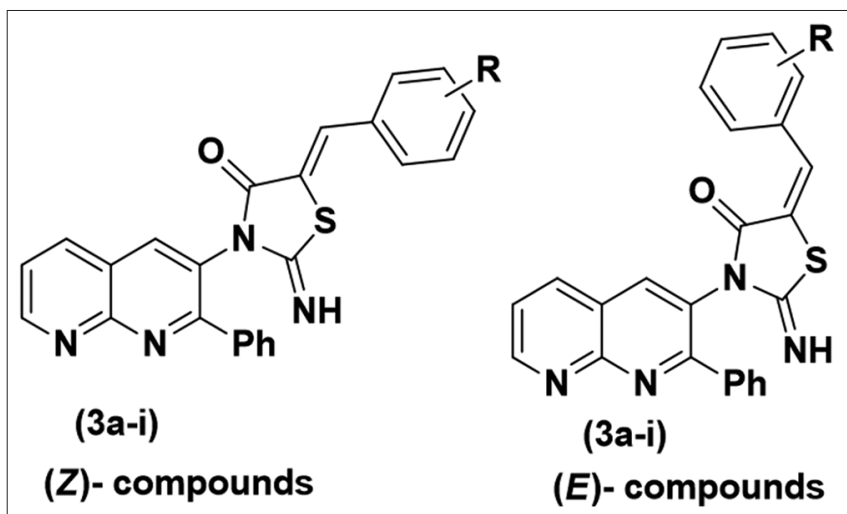

Fig. 3: E/Z isomerism of compounds 3a-i

loaded within the bacterial strain and were incubated at $37^{\circ} \mathrm{C}$ for 24-48 h. After the 24-48 h, the Petri dishes were examined for growth inhibition zone $(\mathrm{mm})$. All determinations were performed in triplicate and the average value was taken for each compound.

\section{CONCLUSIONS}

We have developed a series of 1,8-naphthyridine based arylidene derivatives (3a-i) in good yields. The structures of newly synthesized products were fully studied by elemental and spectroscopic data. The simplicity, easier workability, applicability is reported in this approach. The preliminary results of bioassay demonstrated that the compound $3 \mathrm{~b}$ possess remarkable antibacterial activity against the microorganisms compared with standards.

\section{ACKNOWLEDGMENT}

One of the authors (KR) gratefully acknowledges CSIR-UGC, New Delhi, India, for the award of JRF. We also acknowledge Vice-Chancellor, Mahatma Gandhi University, Nalgonda for providing us with research facilities.

\section{AUTHOR'S CONTRIBUTIONS}

BS (third author), who had wrote the manuscript and submitted same. Remaining authors have added value to it. All authors read and approved the final manuscript.

\section{CONFLICTS OF INTEREST}

The authors declare that they have no conflicts of interest.

\section{REFERENCES}

1. Havrylyuk D, Zimenkovsky B, Vasylenko O, Gzella A, Lesyk R. Synthesis of new 4-thiazolidinone, pyrazoline-, and isatin-based conjugates with promising antitumor activity. J Med Chem 2012;55:8630-41

2. Litvinov VP. Chemistry and biological activities of 1,8-naphthyridines. Russ Chem Rev 2004;73:637-70.

3. Egawa H, Miyamoto T, Minamida A, Nishimura Y, Okada H, Uno H, et al. Pyridonecarboxylic acids as antibacterial agents 4. Synthesis and antibacterial activity of 7-(3-amino-1-pyrrolidinyl)-1-ethyl-6- fluoro-1,4 -dihydro-4-oxo-1,8-naphthyridine-3-carboxylic acid and its analogues. J Med Chem 1984;27:1543-8.

4. Mahesh R, Dhar AK, Jindal A, Bhatt S. Design, synthesis and evaluation of antidepressant activity of novel 2-methoxy 1,8 naphthyridine 3-carboxamides as 5-HT3 receptor antagonists. Chem Biol Drug Des 2014;83:583-91.

5. Badawneh M, Aljamal J. Synthesis and antitubercular activity of piperidine and morpholine 1,8-Naphthyridine analogues. Int J Pharm Pharm Sci 2016;8:252-7.

6. Atanasova M, Ilieva S, Galabov B. QSAR analysis of 1,4-dihydro-4oxo-1-(2-thiazolyl)-1,8-naphthyridines with anticancer activity. Eur J Med Chem 2007;42:1184-92.

7. Badawneh M, Ferrarini PL, Calderone V, Manera C, Martinotti E, Mori $\mathrm{C}$, et al. Synthesis and evaluation of antihypertensive activity of 1,8-naphthyridine derivatives. Part X. Eur J Med Chem 2001;36:925-34.

8. Ferrarini PL, Badawneh M, Franconi F, Manera C, Miceli M, Mori C, et al. Synthesis and antiplatelet activity of some 2,7-di(N-cycloamino)3-phenyl-1,8-naphthyridine derivatives. Farmaco 2001;56:311-8.

9. Mulwad VV, Mir AA, Parmar HT. Synthesis and antimicrobial screening of 5-benzylidene-2-imino-3-(2-oxo-2H-benzopyran-6yl)- thiazolidine-4-one and its derivatives. Ind J Chem 2009;48:137-41.

10. Ottanà R, Maccari R, Barreca ML, Bruno G, Rotondo A, Rossi A, et al. 5-Arylidene-2-imino-4-thiazolidinones: Design and synthesis of novel anti-inflammatory agents. Bioorg Med Chem 2005;13:4243-52.

11. Abo-Salem HM, Ebaid MS, El-Sawy ER, El-Gendy AE, Mandour AH. Synthesis and DPPH radical-scavenging activity of some new 5-( $N$-substituted-1H-indol-3-yl)-5H-thiazolo[4,3-b]-1,3,4-thiadiazole derivatives. Egypt Pharm J 2013;12:11-9.

12. Liu HL, Lieberzeit Z, Anthonsen T. Synthesis and fungicidal activity of 2-imino-3-(4-arylthiazoly-2-yl)-thiazolidinones and their 5-arylidine derivatives. Molecules 2000;5:1055-61.

13. Eman SN, Salwa ME, Eman RZ. Synthesis, anticancer evaluation and molecular modeling of some substituted thiazolidinonyl and thiazolyl pyrazole derivatives. Int J Pharm Pharm Sci 2015;7:353-9.

14. Gursoy A, Terzioglu N. Synthesis and isolation of new regioisomeric 4-thiazolidinones and their anticonvulsant activity. Turk J Chem 2005;29:247-54.

15. Lakhan R, Singh RL. The synthesis and evaluation of 2-imino-3-(4arylthiazol-2-yl)-4-thiazolidinones and their 5-arylidene derivatives as potential agricultural fungicides. Proc Indian Acad Sci Chem Sci 1991;103:33-41.

16. Saravanan G, Selvaraju R, Nagarajan S. Synthesis of novel 2-iminothiazolidin-4-ones. Syn comm 2012;42:3361-7.

17. Ramalingam K, Ramesh D, Sreenivasulu B. Regioselective synthesis of novel [N-(4-oxo-3-(2-phenyl-1,8-naphthyridin-3-yl)-thiazolidin-2ylidene)]acetamide benzamides and their biological activity. Asian J Chem 2019;31:1246-50

18. Ramalingam K, Ramesh D, Sreenivasulu B. Synthesis of 2-[(5-aryl1,3,4-oxadiazol-2-yl)thio]- $N$-(2-phenyl-1,8-naphthyridin-3-yl) acetamide and 2-[substituted-( $1 H$-benzo[ $d$ ] imidazol-2-yl)thio]- $N$-(2phenyl-1,8-naphthyridin-3-yl)acetamide derivatives as antibacterial agents. Rasayan J Chem 2019;12:583-8.

19. Momose Y, Meguro K, Ikeda H, Hatanaka C, Oi S, Sohda T, et al. Studies on antidiabetic agents. X. Synthesis and biological activities of pioglitazone and related compounds. Chem Pharm Bull (Tokyo) 1991;39:1440-5.

20. Hosseinzadeh N, Hasani M, Foroumadi A, Nadri H, Emami S, Samadi N, et al. 5-Nitro-heteroarylidene analogs of 2-thiazolylimino4-thiazolidinones as a novel series of antibacterial agents. Med Chem Res 2013;22:2293-302.

21. Benson HJ. Microbiological Applications. $5^{\text {th }}$ ed. Boston, USA: W.C. Brown Publications; 1990. p. 134. 